\title{
Analysis of the dynamic response of a long span bridge using GPS/accelerometer/anemometer under typhoon loading
}

\author{
Houzeng Han $^{\text {a }}$, Jian Wang ${ }^{\text {a,b }}$, Xiaolin Meng ${ }^{\text {b,c,* }}$, Hua Liu ${ }^{d}$ \\ ${ }^{a}$ School of Environment Science and Spatial Informatics, China University of Mining and Technology, Xuzhou 221116, Jiangsu, China \\ ${ }^{b}$ Nottingham Geospatial Institute, The University of Nottingham, Nottingham NG7 2TU, UK, email: xiaolin.meng@nottingham.ac.uk \\ 'Sino-UK Geospatial Engineering Centre, The University of Nottingham, Nottingham NG7 2TU, UK \\ ${ }^{d}$ China Railway Major Bridge (Nanjing) Bridge and Tunnel Inspect\&Retrofit Co.,Ltd. Nanjing210061, China
}

Abstract

Large flexible engineering structures, such as long span bridges or tall buildings, are susceptible to quasi-static and dynamic deformations caused by different loading situations, thus accurate displacement measurements are desirable to assess the integrity and reliability of the structure. In this study, an integrated system that includes Global Positioning System (GPS), accelerometer and anemometer was developed to obtain the responses of a long span bridge to the extreme wind loading. Spectral analysis based on the Fast Fourier Transform (FFT) algorithm was first carried out to detect the dominant frequencies of the bridge. Then the noisy GPS displacement measurements and accelerometer data are de-noised using the Vondrak filter, and the low frequency disturbance was separated from GPS displacement time series. A least-squares based displacement reconstruction scheme using noise-mitigated accelerations was employed, and the Tikhonov regularization scheme with optimal selected regularization factor was used to alleviate the ill-posedness. At last, an adaptive recursive least squares (RLS) filter was adopted to separate the slow-varying movements, and the total displacement with enhanced measurement accuracy was obtained from the combined quasistatic and high-frequency dynamic displacements. A field monitoring data set collected on the Erqi Yangtze River Bridge, a three-tower cable-stayed bridge located in Wuhan in China, was used to validate the effectiveness of the proposed integration processing scheme. The GPS/accelerometer/anemometer installed on the center supporting tower was used to characterize the interaction between the responses and the ambient wind loadings. The results demonstrate the proposed technique can significantly improve the measurement accuracy of bridge displacement under strong winds. The deformation with the amplitude of several millimeters can be successfully detected, and the spectrum of the bridge response obtained from both GPS data and accelerometer data reveals the identified first natural frequency of the bridge is $0.172 \mathrm{~Hz}$.

Keywords: GPS; accelerometer; anemometer; wind loadings; bridge monitoring; displacement reconstruction; Vondrak filter; recursive least squares (RLS) filter

\section{Introduction}

Bridges play an important role in transportation around the world. An increasing number of modern bridges with longer spans have been built as the development of new construction techniques and the use of new materials. The long span bridges are susceptible to ambient excitations, such as strong winds, earthquakes, traffic loadings and temperature effects, which cause serious concern from bridge engineers. It is vital important to establish a monitoring system that implements an online damage identification procedure.

The Global Positioning System (GPS) has been widely used to monitor the dynamic responses of civil engineering structures, it has the advantage to reliably measure the absolute 3D quasi-static and dynamic displacements. Meng presented the bridge monitoring applications using GPS, and series trials have been conducted on the bridges, including London Millennium Bridge, Forth Road Bridge and Wilford suspension bridge in the UK. The data has been extensively analyzed and the accuracies recorded have been improved over time, starting initially from a few centimeters to what is now a few millimeters [1]. Meo et al. (2006) used GPS to identify the modal parameters including natural frequencies, damping coefficients and mode shapes of a medium span suspension bridge by using the wavelet transforms [2]. Psimoulis et al. (2008) investigated the potential of GPS to identify multi-dominant frequencies of civil engineering structures excited by various loads using least squares-based spectral analysis and wavelet techniques [3]. Nickitopoulou et al. (2006) simulated harmonic movements of slender engineering structures by numerous experiments with a rotation device. It was found that the GPS can monitor displacements with an amplitude larger than $15 \mathrm{~mm}$, at a level of outliers <1.5\% [4]. Breuer et al. (2008) described a procedure using GPS to monitor the deformation of the Stuttgart TV Tower caused by the effects of sun and wind. It indicated that the GPS can measure the total dynamic wind response, not only its dynamic fluctuating component but also the static component [5]. Hristopulos et al. (2007) proposed a systematic procedure for modeling the stochastic and systematic components of the GPS displacements of a tall-building, and the natural frequency of the building was accurately extracted at $0.19 \mathrm{~Hz}$ [6]. GPS can not only measure the dynamic displacements of high-rise buildings, cable-stayed bridges and other flexible structures, it can also be used to monitor stiff civil engineering structures with higher sampling rates [7]. With a sampling rate up to 100 $\mathrm{Hz}$, the high-rate GPS receiver could be used not only to measure the deflections, but also to determine the high frequencies of the bridges $[8$, 9]. The noise characteristics of the high-frequency GPS measurements have been illustrated by several researchers [10-12]. With the development of current Global Navigation Satellite System (GNSS) technology, the dynamic displacements can be identified with higher accuracy using the multimode GNSS data [13].

For a real-time monitoring system, it requires on-the-fly ambiguity resolution independent of receiver's dynamics. The improved ambiguity resolution performance can be obtained with the modernized triplefrequency GPS [14]. However, GPS alone would not be able to provide highly accurate and robust monitoring of bridges due to inherent limitations of GPS such as multipath, uncorrected tropospheric delay, satellite visibility conditions and heavy reliance on communications for real time applications [15]. In addition, the accuracy of height component is usually 2-3 times worse than the horizontal component. Among which, the multipath effect becomes the major error source for short-baseline high accuracy deformation monitoring applications. Many multipath mitigation strategies have been proposed in recent years, including the hardware-based methods $[16,17]$ and software-based methods $[18,19]$. It has been found that the sidereal filtering is an easy method that can be used to remove the multipath effects [20, 21]. However, in real deformation monitoring applications, dynamic multipath effects change with time due to the changing of the geometry of satellites and reflective surfaces [22]. A real-time multipath effects correction model is still absent.

For a comprehensive monitoring system, multi-sensor are usually deployed at the monitoring sites. Pseudolite augmented monitoring 
system is an alternative to improve the geometric strength for GPS monitoring system in constraint conditions [23]. The integrated use of new satellite constellations such as BeiDou, and Galileo are expected to improve the reliability and accuracy of the positioning results. Traditionally, accelerometers are widely applied to measure the response of large structures and can capture very high frequency dynamics because of high sampling rates (up to $1000 \mathrm{~Hz}$ ). The deficiency of an accelerometer for detecting long span bridge deformation is insensitive to long-period movements. As such, the mean component cannot be reliably obtained from the accelerometer. Accelerometer-derived displacements are contaminated by the "drift" problem induced by double integration [24]. In most cases the initial position and velocity information are not available, Park et al. (2005) presented an initial velocity estimation method for bridge displacement determination to overcome these problems [25]. Lee et al. (2010) and Hong et al. (2010) presented a dynamic displacement reconstruction scheme from measured acceleration based a finite impulse response (FIR) filter, which avoids the double integration procedure [26, 27].

Due to the complementary characteristics of GPS and accelerometer in monitoring structural deformation [28], the integrated solution of using GPS and 3D accelerometer is widely deployed [29]. The GPSderived displacements are in close agreement with accelerometer results, which improves the redundancy of the monitoring system [30]. Optimal integration technique can improve the measurement accuracy of reconstructed displacement. Chan et al. (2007) proposed an integrated GPS-accelerometer data processing technique based on the empirical mode decomposition (EMD) and adaptive filter techniques [31]. Smyth et al. (2007) presented a multi-rate Kalman filtering approach to integrate GPS and accelerometer data at different rates [32], Hwang's research shows the frequency-based displacement extraction method was most appropriate for the GPS/accelerometer data integration [33]. However, a tightly coupled integration of GPS and accelerometer for online monitoring has not yet been developed.

This paper introduces an integration algorithm that is used to reconstruct the quasi-static and dynamic displacements of a long-span bridge under strong wind loading. The natural frequencies are extracted using the peak-picking approach based on an FFT algorithm. The frequencies detected from GPS displacement are validated by the spectrum of accelerometer data. Except from detecting the natural frequencies, it is found when tall bridge tower is subjected to ambient wind loadings very slow movements with high amplitude are measured with GPS. However these movements are not multipath effects. To isolate noisy component, the Vondrak filter is applied to de-noise the GPS/accelerometer measurements. The interaction between wind speeds and responses are investigated, the suspected low frequency noises are removed from filtered GPS time series by the spectra comparison with wind speed data [very confusion express: the previous sentence said this slow movement is NOT multipath effect but here you say it is NOISE!]. The optimal total displacements are reconstructed with the recursive least squares (RLS) filter. The effectiveness of the integrated data processing algorithm was evaluated with a field monitoring data set collected on the Erqi Yangtze River Bridge in Wuhan, China.

\section{The SHM system of the Erqi Yangtze RiverBridge in Wuhan}

\subsection{The Erqi Yangtze River Bridge}

The Erqi Yangtze River Bridge is a highway bridge over the Yangtze River in Wuhan, China (Fig.1). The bridge is the world's largest threetower cable-stayed bridge with a bridge length of $2,922 \mathrm{~m}$ The two main spans are $616 \mathrm{~m}$ long each and its pylons are $209 \mathrm{~m}$ high. This bridge carries four lanes of traffic on the Wuhan Second Ring Road in each direction. The construction of the bridge started in 2008 and completed on 31 December 2011.

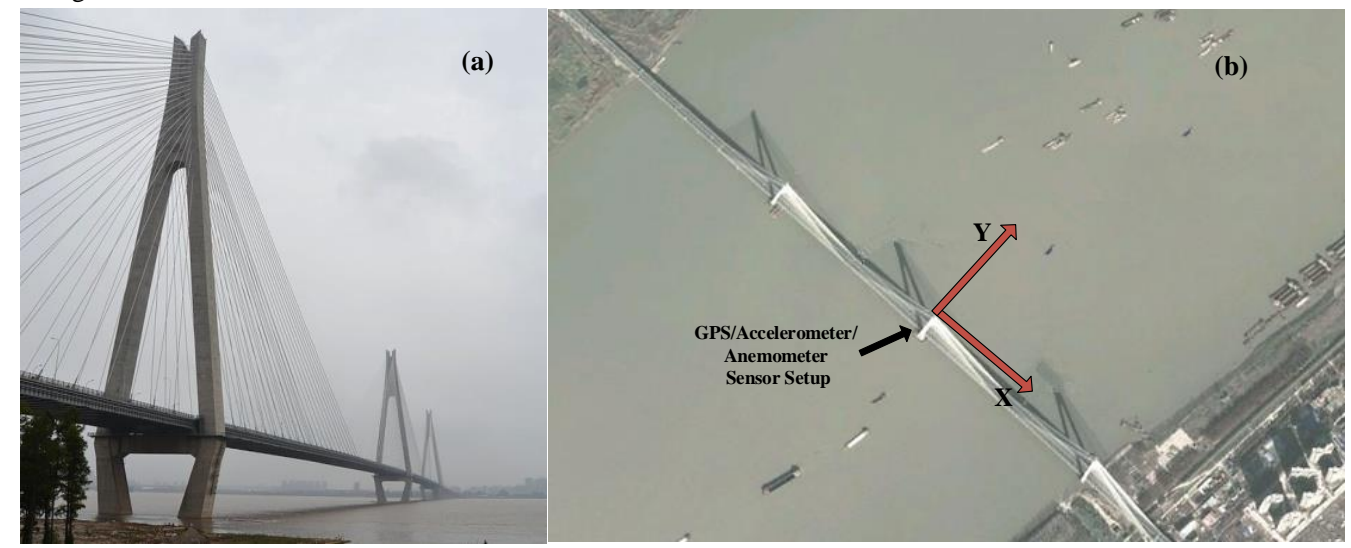

Fig.1.a) The view of the Erqi Yangtze River Bridge in Wuhan (http://en.wikipedia.org/wiki/Erqi_Yangtze_River_Bridgeb) the local bridge coordinate system (BCS).

\subsection{The deployment of the SHM sensors}

The bridge has to stand a variety of loadings that include wind temperature and urban traffic. . In order to evaluate the integrity and reliability of the bridge an effective way is to establish a real-time structural health monitoring (SHM) system. The SHM system of Erqi Yangtze River Bridge consists of structure response monitoring sensors (GPS, accelerometer, displacement transducer, and clinometer), environment monitoring sensors (anemometer, thermometer, and hygrometer) and traffic safety monitoring sensors (HD video camera). The bridge engineers have paid great attention to study the wind-resistant performance, anti-seismic performance, temperature shrinkage effect of the long span bridge with the multi-sensors systems.
In this paper, we present an integrated deformation monitoring system that consists of GPS, accelerometer and anemometer. The dynamic displacements and modal frequencies, which act as key indicators of bridge structural damage, will be extracted more accurately with the data strategy developed by the authors. A particular attention is paid to assess the bridge performance under strong wind loadings.

The instrument set-up for the bridge deformation monitoring can be seen in Fig.2. It consists of three dual-frequency NovAtel GNSS receivers (A, B and C) with a sampling rate of $10 \mathrm{~Hz}$ deployed on three towers. The other two GNSS receivers (D and E) were installed on the main span of the bridge. Choke-ring antennas were used to mitigate multipath effect induced by the cables, towers and passing vehicles. One reference station was installed on the roof of a nearby office building. 
The baselines are less than $20 \mathrm{~km}$. [The height difference among the receivers CANNOT be less than $10 \mathrm{~m}$ !] The displacement time histories were obtained by processing the GPS data in a real time kinematic (RTK) manner, and the original displacements in WGS 84 were first converted to the bridge coordinate system (BCS) frame. The accuracy and availability of GPS deformation monitoring system is subjected to the open sky conditions, during the trial, more than seven available GPS satellites can be tracked most of time (Fig. 3), which guaranteed the GPS positioning performance.

A total of seven triaxial accelerometers were also rigidly fixed at monitoring sites of interest, the body frame of the accelerometers were carefully aligned to the BCS, whose $x$ axis is parallel to the longitudinal direction of the bridge, $z$ axis points towards vertical direction and $y$ axis completes a right-handed coordinate system (Fig.1). The raw accelerometer data was collected at the rate of $20 \mathrm{~Hz}$, precise time synchronization between GPS and accelerometer was conducted. The accelerometers were expected to monitor the high-frequency response of the bridge in real time, and accelerometer measurements are used to detect the modal frequencies of the bridge.

In addition to GPS and accelerometers, three anemometers were also installed on the tower roofs to capture the wind effect, the wind speed data was recorded at $1 \mathrm{~s}$ sampling interval. All data sets were recorded and stored on disk.

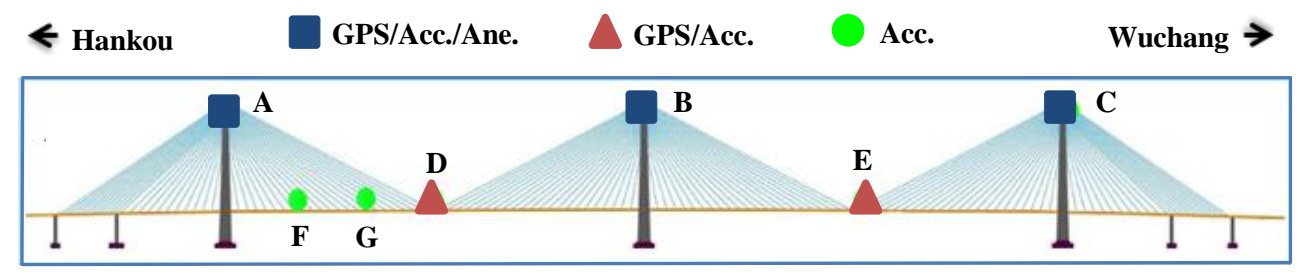

Fig.2. Instrument setup for the Erqi Yangtze River bridge deformation monitoring system.

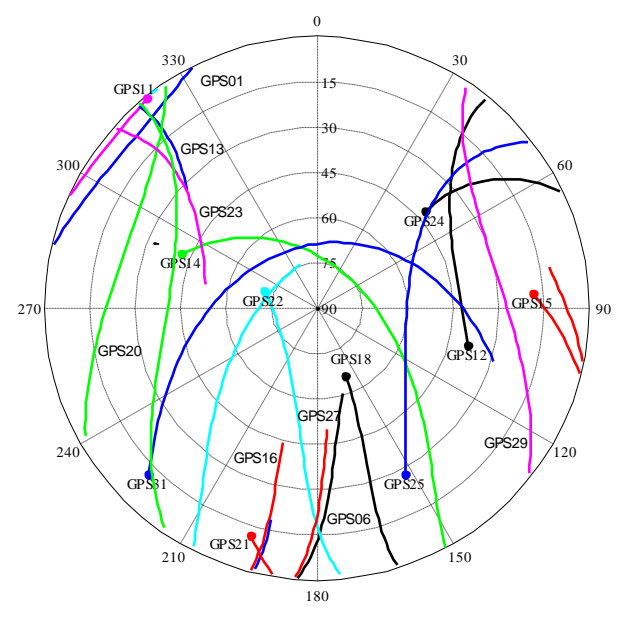

Fig.3.6h skyplot of the visible satellites at site B during the test.

\section{Field data collection using GPS/accelerometer/anemometer under typhoon loading}

The filed bridge monitoring data was collected on August 18,2013 , when a typhoon occurred in the region of Wuhan, the maximum wind speed peaked at $46.5 \mathrm{~m} / \mathrm{s}(167.4 \mathrm{~km} / \mathrm{h})$, the bridge SHM system has recorded the wind loadings and related structural response information.

However, in this research, only the GPS/accelerometer/anemometer data sets collected at Site B, which is expected to experience the strongest dynamic response during wind gust, are used as a demonstration of the dynamics reconstruction and modal frequencies detection using the proposed integration scheme, the whole data sets lasted for ten minutes (64940s to 655400 s, GPS week: 1754), the bridge response were mainly caused by the strong wind gust.

In this section, the results of dynamics reconstruction and frequencies detection using the integration approach will be demonstrated in detail, only the bridge's response to wind loadings in the horizontal direction has been analyzed.

Fig.4shows the time series for the GPS-derived displacements forlongitudinal movements, the corresponding acceleration time series, and wind speed. The lateral response measurements are shown
inFig.5.As mentioned previously, the bridge generally experienced ambient strong wind loadings during the data collection period, the bridge movements contain a typical long-period component, which are indicated by a red line in Fig.4 and Fig.5,the signal decomposition is based on the moving average filter with a window size of $5 \mathrm{~s}$ (50 epochs).Wind-induced response of thetall bridge tower comprises a quasi-static component and a high-frequency dynamic fluctuating component.

It is evident these very low movements of several centimeters are generally not multipath effect, and the natural frequency band of the bridge is actually above the very low frequency range. It is also verified by analyzing the cross correlations between the horizontal displacements and wind speed data during the observation period (Fig. 6), the best fitting curves are obtained by using the least square method to find a quadratic function that best matches the data, it can be seen the bridge horizontal movements have high correlation with the wind speed, the cross correlation coefficient between the longitudinal movements and wind speed reaches 0.65 , while a higher cross correlation coefficient with 0.83 can be obtained between the lateral movements and wind speed. The remaining short-period component of the apparent displacements is mainly composed of background noise and the high-frequency response.

We can also see that the acceleration time series with a sampling rate of $20 \mathrm{~Hz}$ also contains noisy component, so it is better to eliminate the noise component with a specific low-pass filter before displacement reconstruction. 

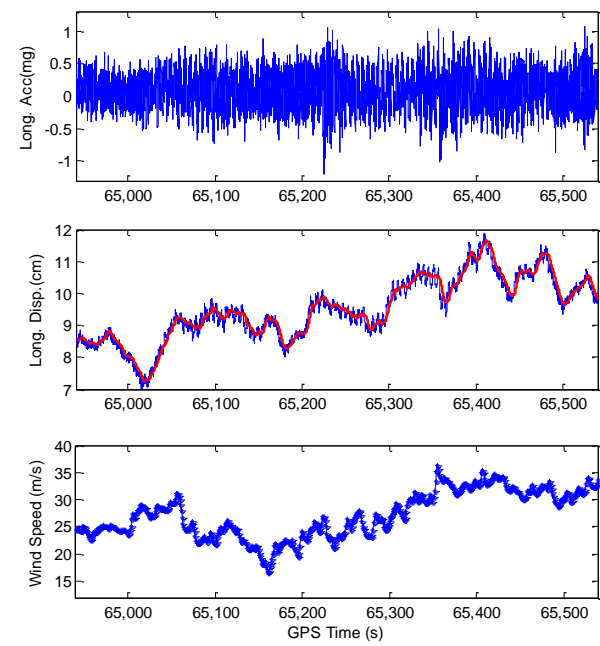

Fig. 4. Time series for acceleration (top), GPS displacement (middle) and wind speed (bottom) in longitudinaldirection.
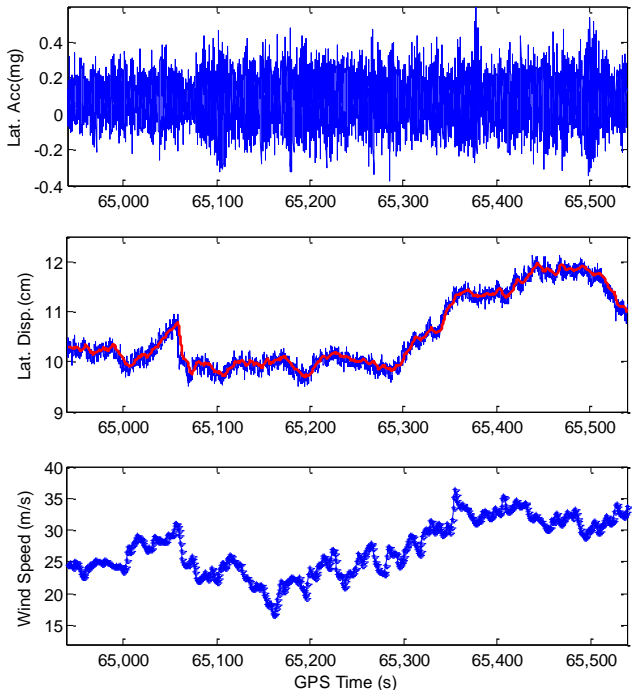

Fig. 5. Time series for acceleration (top), GPS displacement (middle) and wind speed (bottom) in lateral direction.
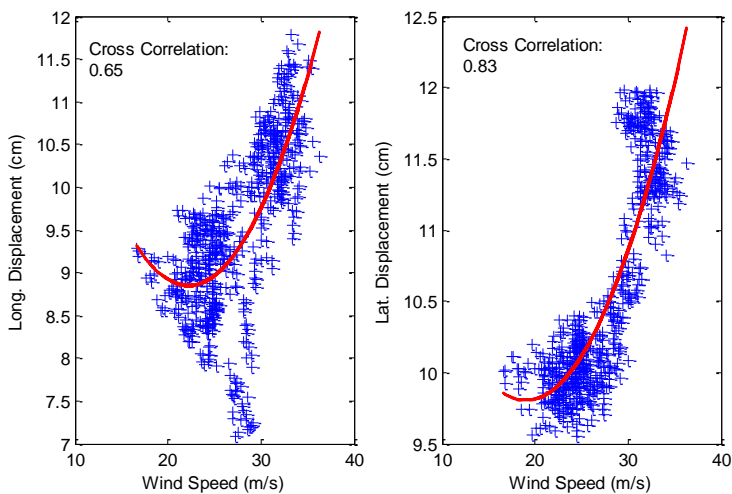

Fig. 6. The cross correlation between wind speeds and GPS displacements.

\section{Anemometer aided GPS dynamic deflection extraction}

The raw GPS displacement measurements are initially processed using the embedded software in real-time, and the anemometer and accelerometer are operated parallelly. The GPS can be used successfully to capture the quasi-static and dynamic movements, however the GPS measurements are generally suffered from multipath effects and measurement noise, as such measurement disturbance will decrease the applicability of GPS for high accuracy monitoring applications. In this paper, the Vondrak filter is utilized to remove the noise-contaminated GPS time series.

\subsection{Vondrak filter}

Vondrak filter method is an effective smoothing method that aims to compromise between two extreme circumstance, namely absolute smoothing $(\mathrm{S})$ and absolute fitting $(\mathrm{F})$, and it does not require any predefined fitting function, the objective function of Vondrak filter can be expressed as follows[20, 34]

$$
Q=F+\lambda^{2} S \rightarrow \min
$$

with

$$
\begin{aligned}
& F=\sum_{i=1}^{n} p_{i}\left(\tilde{y}_{i}-y_{i}\right)^{2} \\
& S=\sum_{i=1}^{n-3}\left(\Delta^{3} \tilde{y}_{i}\right)^{2}
\end{aligned}
$$

where $\tilde{y}_{i}$ is the smoothed value corresponding to the original measurement $y_{i}, p_{i}$ is the weight of $y_{i}, \Delta^{3} \tilde{y}_{i}$ is the third-order differentiation of the smoothed values, and $\lambda^{2}$ is a positive parameter used to adjust the degree of smoothness of the filtered time series $\tilde{y}_{i}$.

When applying Vondrak filter in practice, the smoothing factor $\mathcal{E}$, where $\varepsilon=1 / \lambda^{2}$, should be carefully selected, it is obviously stronger smoothing effect can be obtained with smaller $\varepsilon$,the frequency response method was applied to determine the optimal smoothing factor in this paper. In fact, Vondrak filter is applicable to extract both short-term and long-term components from original measurements when using an optimal smoothing factor.

In addition, Vondrak filter has the advantage of the applicability to process both equal and non-equal non-linear data series over other filtering method, which makes it an appropriate filter method for deformation applications. For the particular GPS/Accelerometer integrated deformation monitoring applications, Vondrak low-pass filter can be used to separate the displacement signal and acceleration signal from measurement noise, on the other hand, the multipath signature, which is the most important error source that deteriorates the accuracy of GPS deformation monitoring, can also be filtered out using the Vondrak band-stop filter considering the suspected multipath frequency band.

\subsection{Dynamic deflection extraction}

First, the data was collected using GPS/accelerometer/anemometer deployed on the specific bridge, and the data from different sensors must be synchronized before integration, the GPS displacement time series are originally defined in the ECEF frame, namely WGS84 coordinate system, to make integration with accelerometer data, transformation of GPS ECEF coordinates into the local bridge coordinate system (BCS) is required. Data gaps recovery and outliers rejection procedure should also be applied.

Second, the modal frequencies of the bridge are extracted from GPS displacement data and accelerometer data using the Fast Fourier Transform (FFT) algorithm, the spectrum of the anemometer data is also obtained by the FFT, the time-frequency comparison between GPS data and anemometer data is conducted, and the suspected frequency band of GPS multipath signature is determined.

Third, the Vondrak filter is applied to GPS data to remove the high- 
frequency measurement noise, the cut-off frequencies are determined from FFT analysis, and low frequency noise in the GPS data series has also been filtered out by Vondrak filter, thus 'clean' displacement time series have been obtained.

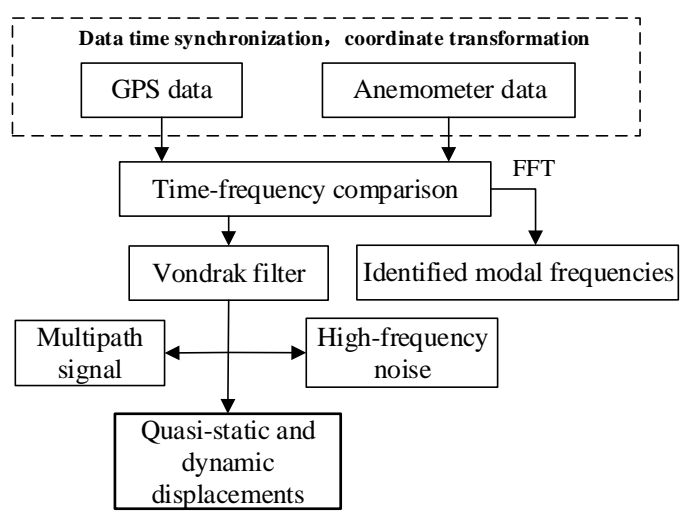

Fig.7.Processing GPS data by Vondrak filter
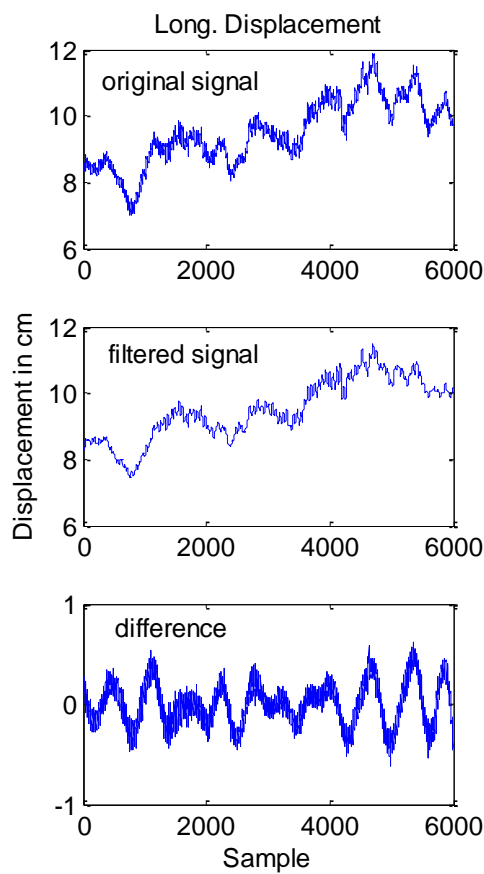

\subsection{Field data analysis}

Spectra analysis on GPS data reveals that significant movements derived from GPS are confirmed to a band below $0.3 \mathrm{~Hz}$ for both longitudinal displacements and lateral displacements (which will be illustrated in more detail in Section 6.3), the displacements beyond this scope are characterized by measurement noise, which should be removed by the low-pass filter.

In this research, the Vondrak filter as explained in Section 4.1 will be used, the optimal smoothing factor was selected using the frequency response method. Fig. 8 shows the typical results of the Vondrak filter applied to the displacement time series, the high-frequency noise and multipath effects have been filtered out, it can be seen that the amplitude of the noise component is less than $1 \mathrm{~cm}$, and different noise characteristics are obtained for longitudinal displacement and lateral displacement, it seems that the multipath effects affecting GPS mostly along longitudinal direction. The filtered GPS time series are then used as desired signal during the RLS filter process to obtain the total displacements, meanwhile the original accelerometer data sets are also de-noised using the Vondrak filter and then used to reconstruct displacements.
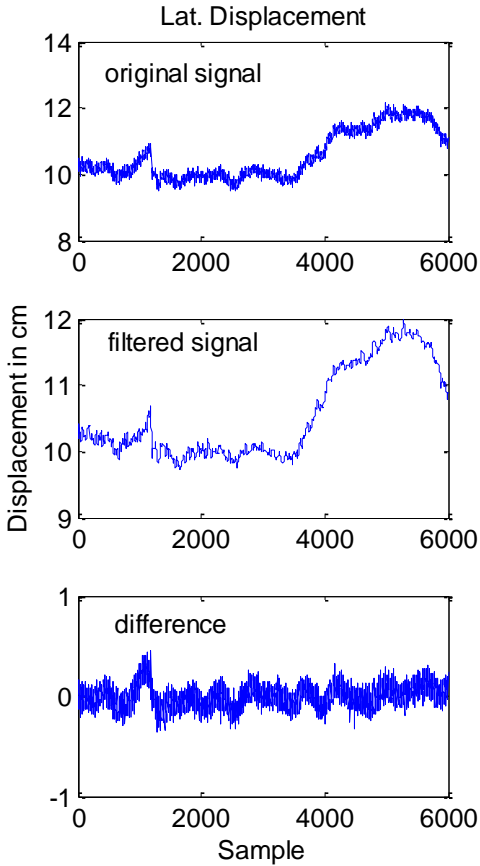

Fig. 8. GPS displacement time series (top); Vondrak filtered GPS signal (middle); difference between original signal and filtered values (bottom)

\section{Accelerometer-derived displacement reconstruction}

5.1 High frequency acceleration integration based on Tikhonov regularization algorithm

For the bridge deformation monitoring applications, the dynamic displacements can be obtained from RTK GPS, but the positioning results are usually very noisy which are contaminated by measurement noise and multipath interference. Traditionally, the accelerometer is widely used to identify the dynamic parameters of the structure, however the deficiency of accelerometer for bridge monitoring is the "drift" problem of acceleration-derived displacement induced by double numerical integration, and only relative displacement can be obtained. Hong introduced an FIR filter to reconstruct the displacements from acceleration $[26,35]$.The acceleration can be derived fromd is placement data by applying the central differencing scheme, the calculated acceleration $(a)$ in the discrete form can be expressed as

$$
a_{k}=\frac{s_{k+1}-2 s_{k}+s_{k-1}}{(\Delta t)^{2}},(k=1,2, \ldots, n)
$$

where $S_{k}$ is the measured displacement at epoch, $\Delta t$ is the sampling interval.

In the entire time window, the matrix formof Equation (4) can be described as

$$
A X=L+\Delta
$$

$$
\text { with } \boldsymbol{A}=\frac{1}{(\Delta t)^{2}}\left[\begin{array}{ccccccc}
1 & -2 & 1 & & & & \\
& 1 & -2 & 1 & & & \\
& & & \vdots & & & \\
& & & 1 & -2 & 1 & \\
& & & & 1 & -2 & 1
\end{array}\right]_{n \times(n+2)} \quad \boldsymbol{X}=\left[\begin{array}{l}
s_{0} \\
s_{1} \\
\vdots \\
s_{n} \\
s_{n+1}
\end{array}\right] \text {, }
$$




$$
\boldsymbol{L}=\left[\begin{array}{l}
a_{1} \\
a_{2} \\
\vdots \\
a_{n-1} \\
a_{n}
\end{array}\right], \Delta \text { is the accelerometer measurement noise. }
$$

The displacement series can be estimated based on the least square (LS)principle

$$
\min _{\hat{\boldsymbol{X}}} \Pi(\hat{\boldsymbol{X}})=\|\boldsymbol{A} \hat{\boldsymbol{X}}-\boldsymbol{L}\|^{2}
$$

where $\hat{\boldsymbol{X}}$ is the estimated displacement vector.

Due to the rank deficiency problem, the Equation (6) cannot be applied directly, the Tikhonov regularization scheme[36] was adopted to derive the displacement

$$
\min _{\hat{\boldsymbol{X}}} \Pi(\hat{\boldsymbol{X}})=\|\boldsymbol{A} \hat{\boldsymbol{X}}-\boldsymbol{L}\|^{2}+\alpha^{2}\left\|\hat{\boldsymbol{X}}-\boldsymbol{X}^{*}\right\|^{2}
$$

where $\boldsymbol{X}^{*}$ is the static displacement vector, $\alpha$ is the regularization factor.

The estimated displacement can be expressed as

$$
\hat{\boldsymbol{X}}=\left(\boldsymbol{A}^{\mathrm{T}} \boldsymbol{A}+\alpha^{2} \boldsymbol{I}\right)^{-1} \boldsymbol{A}^{\mathrm{T}} \boldsymbol{L}
$$

where $\boldsymbol{I}$ is the identity matrix.

An optimal selection of the regularization factor will play a critical role in the displacement reconstruction step, the L-curve method is applied in this paper to determine $\alpha$ [37]. In order to avoid the unreliable parameters induced by the L-curve method, a rejection-region is set based on priori information to remove the false value, when the number of valid parameters reaches the default value, the final regularization factor is determined by smoothing the valid parameters.

From a practical perspective, the time window size is selected as two to three times of the longest period of the structure based on Nyquist frequency, the coefficient which multiplies the longest period to obtain the optimal windows size is defined as $N_{w}$, and in order to avoid the boundary inaccuracy, only the middle point of the reconstructed displacement is used.

The flowchart of displacement reconstruction from acceleration based on Tikhonov regularization algorithm is shown in Fig. 9. The raw acceleration time history is de-noised by the Vondrak filter and the modal frequencies are also detected by FFT, the initial position and velocity measurements are provided by GPS data, and Tikhonov regularization scheme is applied to solve the rank-deficient problem, the regularization factor is selected byL-curve method with a rejection-region.

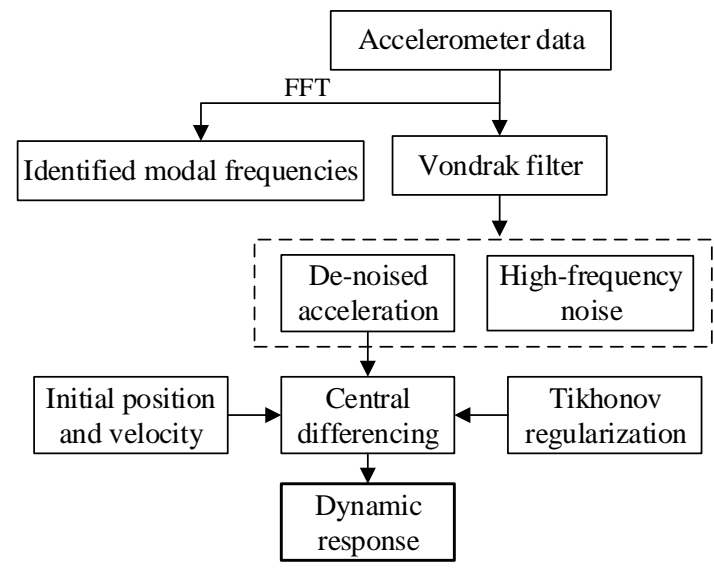

Fig. 9.High frequency acceleration integration based on Tikhonov regularization algorithm

\subsection{Trial analysis}

The accelerations are measured at the rate of $20 \mathrm{~Hz}$, which is expected to contain more high-frequency responses. In this paper, two schemes are designed to reconstruct displacement from the noise-mitigated accelerations, Scheme 1 -displacement reconstruction based on Tikhonov regularization, which was introduced in Section 5.1, Scheme 2 - double integration of the accelerometer-measured accelerations associate with moving average filter. The longitudinal accelerations are used as a demonstration for displacement reconstruction.

Based on the spectra analysis on accelerometer data, the lowest dominant frequency, namely $0.172 \mathrm{~Hz}$, corresponds to fundamental frequency of the bridge. The optimal window size is thus selected as $14.55 \mathrm{~s}$ (the number of data points is 291) considering the lowest dominant frequency, that is $N_{w},=2.5$, the optimized windows size will achieve more accurate displacement estimates, however the efficiency of the approach will be significantly decreased if the window contains too many data points. The optimal regularization factor is determined using the L-curve method considering the rejection region, then the optimal value is selected as $\alpha=0.001$.

On the other hand, a direct double integration of acceleration yields an obvious drift, such a drift, no matter whether it is positive or negative, will significantly deteriorate the accuracy of the reconstructed displacements during short period. An alternative method to remove these drift errors is applying the high-pass filter, the moving average filter was used in this paper. The selection of window size parameter is a major difficulty to obtain optimal displacement estimates, a window of 50 data points was used in the present study based on our experience.

Fig. 10 shows the results of the displacement reconstruction during the wind excitations with the two displacement reconstruction schemes. As shown in the figure, the dynamic displacements reconstructed from two schemes agree with each other, while the displacements extracted from scheme 2 show obvious deviation for a particular time period, and it also produces some abnormal errors which bias the displacement estimates, and the amplitude of the displacements derived from scheme 2 are slightly smaller than that of displacements derived from scheme 1 . The results indicate more exact displacements can be reconstructed from scheme 1 , it also can be seen that accelerometer is suitable to measure the deformations with small amplitude accurately.

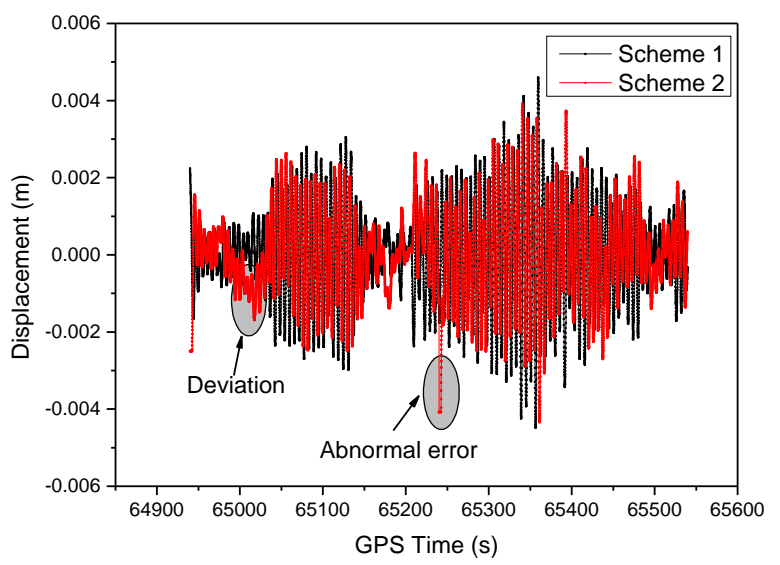

Fig. 10. Reconstructed longitudinal displacement using Tikhonov regularization approach and double integration approach.

\section{The schematic for extracting of the dynamic responses of the Bridge}

During the strong wind excitations, the displacement response of the large bridge tower can be regarded as the sum of quasi-static 
displacements and a dynamic component. The optimal displacements of the structure can be reconstructed from GPS displacements and accelerometer-derived displacements using the recursive least squares adaptive (RLS) filter.

\subsection{Recursive least squares adaptive filter}

An adaptive filter can be used to separate the coherent component from two measurement inputs, namely reference measurement and desired signal. The adaptive RLS filter is an efficient algorithm that performs an exact minimization of the sum of the squares of the desired signal estimation errors at each time step. The RLS algorithm can be summarized as follows[38]

$$
u(k)=\boldsymbol{f}^{T}(k) \boldsymbol{w}(k-1)
$$

where $u(k)$ is the filter output at step $k, \boldsymbol{w}(k-1)$ is the filter weights,

$\boldsymbol{f}(k)$ is the input history vector.

The estimationerror $e(k)$ can be described as

$e(k)=d(k)-y(k)$

where $d(k)$ is desired signal.

The gain vector $\boldsymbol{g}(k)$ is calculated by the following equation

$$
\boldsymbol{g}(k)=\frac{\boldsymbol{R}^{-1}(k-1) \boldsymbol{f}(k)}{\mu+\boldsymbol{f}^{T}(k) \boldsymbol{R}^{-1}(k-1) \boldsymbol{f}(n)}
$$

with the covariance $\boldsymbol{R}(k)$ given by

$$
\boldsymbol{R}(k)=\mu^{-1}\left[\boldsymbol{R}^{-1}(k-1)-\boldsymbol{g}(n) \boldsymbol{f}^{T} \boldsymbol{R}^{-1}(k-1)\right]
$$

where $\mu$ ( 0 to 1 ) is called forgetting factor, commonly used values are between $0.95<\mu<0.995$, a variable forgetting factor is determined in this paper[39].

The filter weights is updated for the next step

$$
\boldsymbol{w}(k)=\boldsymbol{w}(k-1)+\boldsymbol{g}(k) e(k)
$$

The RLS filter determines the optimal filter weights on a step-by-step basis, for the stationary signals, the filter weights converge to steady values, however, they will keep change during the variation of the process for non-stationary signals.

The RLS filter performance is superior to the conventional least mean squares (LMS) algorithm, and its performance is hardly restricted by the abnormal errors occurred in the desired time series. On the other hand, the convergence speed of RLS filters is faster than LMS filter, that is, RLS filter is more suitable for real-time monitoring applications, especially when the abrupt changes happen.

\subsection{Integrated deflection extraction procedure}

The overall flowchart of the processing procedure to extract bridge dynamics and modal frequencies using GPS/accelerometer/anemometer integrated data is provided in Fig.11,the bridge responses induced by the strong wind gust consist of static or quasi-static movements and dynamic components, and the total displacement responses can be measured with the multi-sensor integration system.

First, the RLS filter is used to obtain the low-frequency bridge response. The de-noised time history from GPS data is used as desired signal, the high-frequency dynamic displacement time history derived from accelerometer data is considered as reference measurements. The resulting uncorrelated component is the low-frequency dynamic displacement.

Second, the total bridge dynamics is reconstructed from the combination of low-frequency dynamic displacements derived from RLS filter and the high-frequency dynamic components given by the acceleration-derived displacement.

Third, the FFT algorithm is then used to extract the modal frequencies from the integrated displacements.

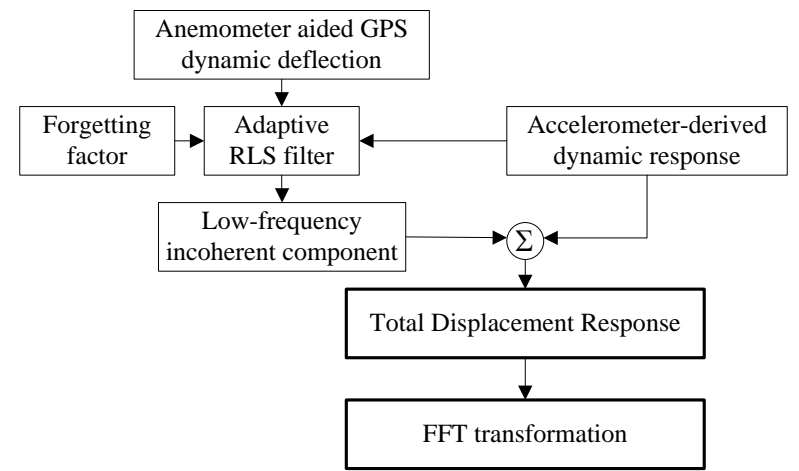

Fig.11.Proposed algorithm to extract bridge dynamics and modal frequencies from GPS/accelerometer.

Previous analysis reveals that accelerometer alone is unable to measure the quasi-static movements, furthermore, GPS alone cannot measure the low-frequency movements and dynamic movements accurately, especially for the high-frequency dynamics. In order to obtain the optimal displacements from GPS and accelerometer, the RLS filter isapplied to extract total displacements. The filtered displacement time history from GPS is taken as the desired signal, while accelerationreconstructed displacements act as the reference signal, the filter length is set as 6, and a variable forgetting factor is determined. As has been noted in Section 3, the GPS and accelerometer were sampled at different rates, thus a data interpolation process should be applied to GPS filtered data before performing the RLS filter, the spline interpolation method provided by the software MATLAB was used in this study, and the mean displacement has been removed from the filtered signal.

The results of the RLS filter are shown in Fig.12, the coherent and incoherent component are successfully isolated and displayed in the bottom subfigure of Fig.12. The coherent component is regarded as dynamic displacements, while the incoherent component is the lowfrequency movements. It can also be seen less accurate results are obtained during the initial phase, which is due to inaccurate boundary estimates caused by the reconstruction algorithm, another reason is that the RLS filter is still in the transition period. 

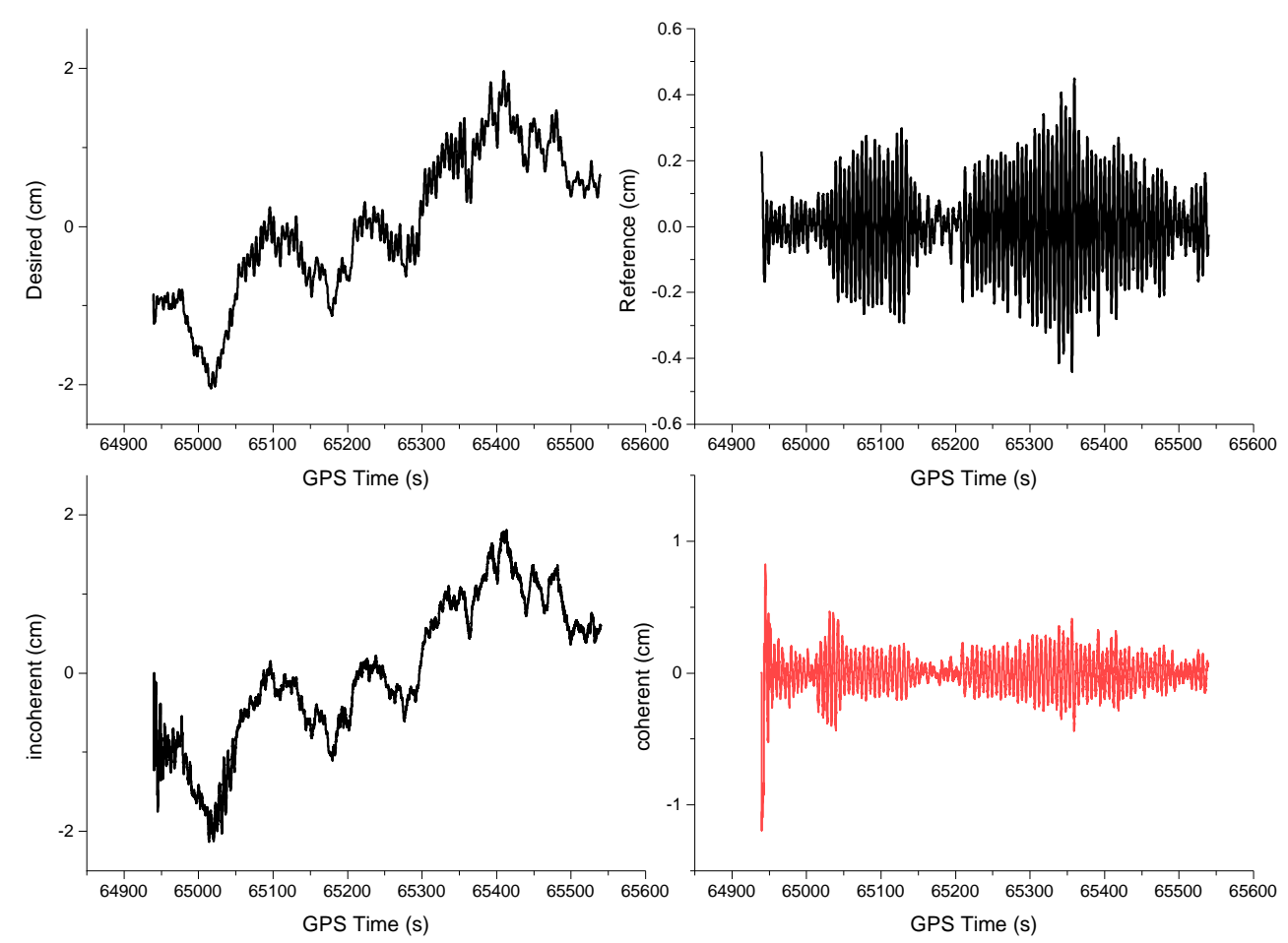

Fig. 12. RLS filter results fromlongitudinalGPS displacements and acceleration reconstructed displacements.

Fig.13 gives the convergence process of the filter weights, it can be seen that RLS filter has a relatively fast rate of convergence to obtain optimal weights, for the non-stationary signals, the filter weights are adjusted on a step-by-step basis, and the filter tracks the variation of the process.

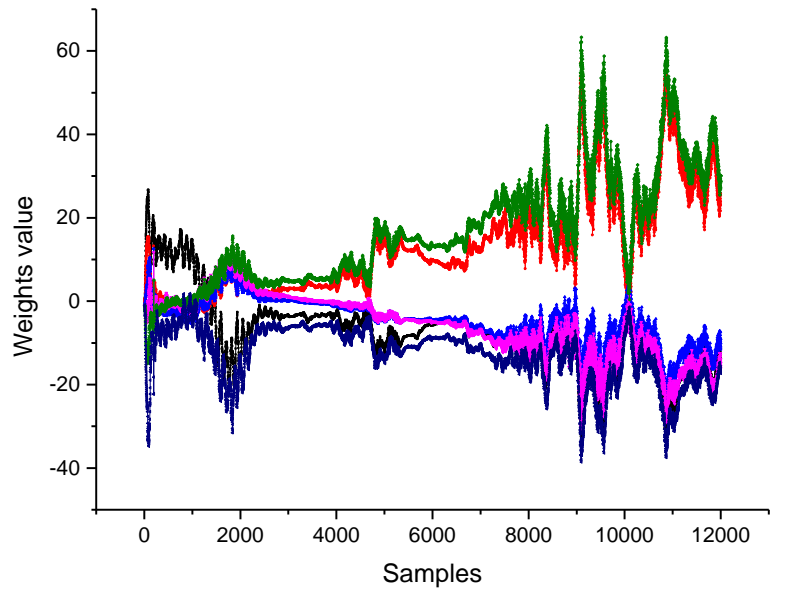

Fig. 13. Estimated weights convergence
The corresponding RLS filter results for the lateral displacement are displayed in Fig.14, as shown in the figure, a noise-like high-frequency displacements with smaller amplitude are reconstructed from lateral accelerations, which means the lateral accelerometer are significantly contaminated by the high-frequency noise, and the correlation between lateral accelerometer-derived displacements and GPS displacements is lower than that of longitudinal component.

After applying the RLS filter, the low-frequency displacements and high-frequency displacements are obtained, respectively. The total displacements are the combination of the two displacement components which are plotted in Fig.15, it can be noted the proposed integrated data processing algorithm can capture both quasi-static and high-frequency responses accurately.Fig. 16 presents the bridge movements in the horizontal direction, the epoch-based movement occurs in Fig.16 as irregular tracks with long-axis in longitudinal direction $(4.2 \mathrm{~cm})$ and a shorter axis in lateral direction $(2.5 \mathrm{~cm})$, the change of the movements are induced by the wind loadings. It can be concluded that the proposed algorithm can accurately measure the multipath-free quasi-static responses induced by strong wind loading, at the same time, the highfrequency dynamic movements can also accurately captured, this will benefit to ensure the bridge safety in extreme weather conditions. 


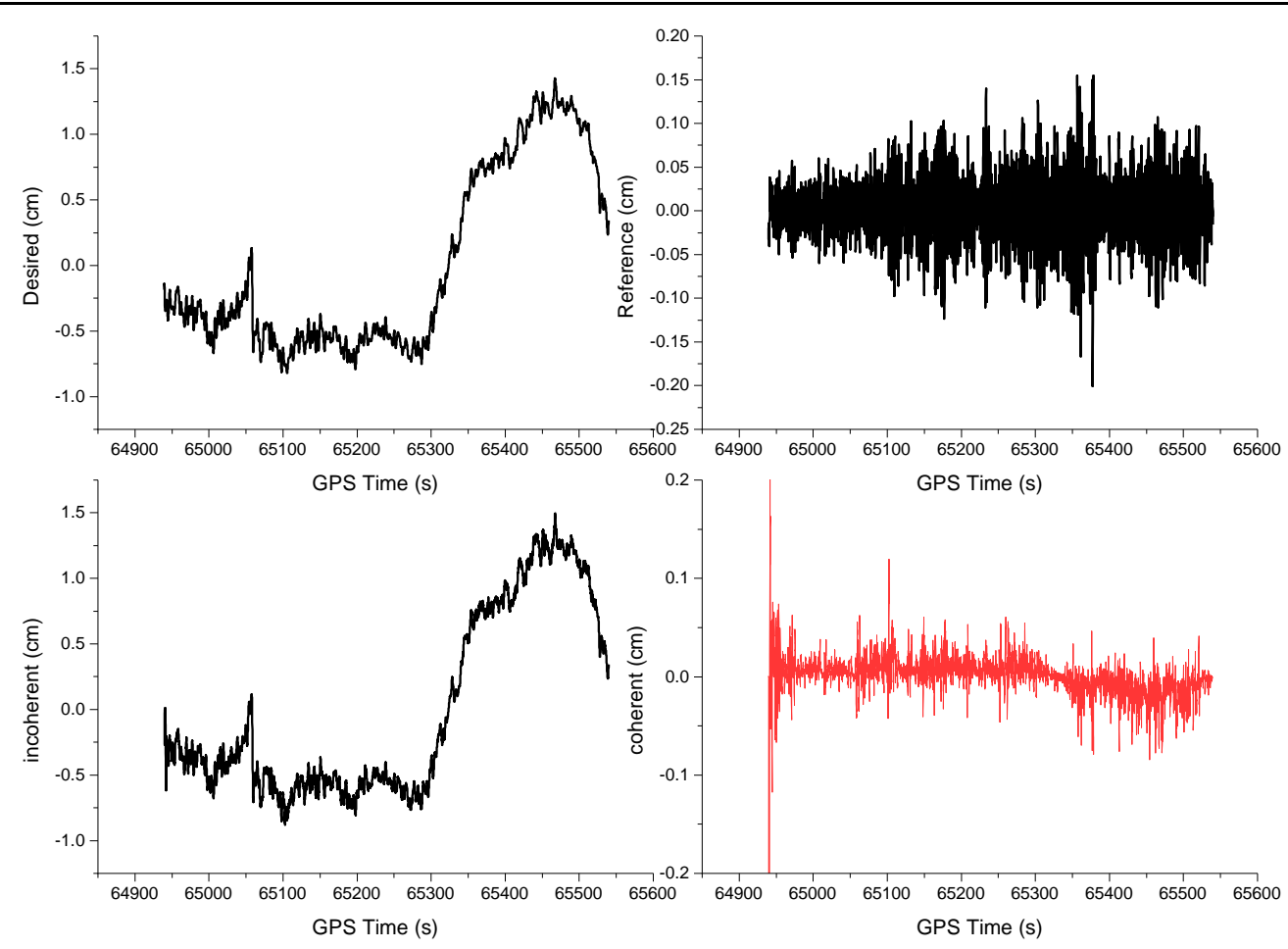

Fig. 14. RLS filter results fromlateral displacements and acceleration reconstructed displacements.

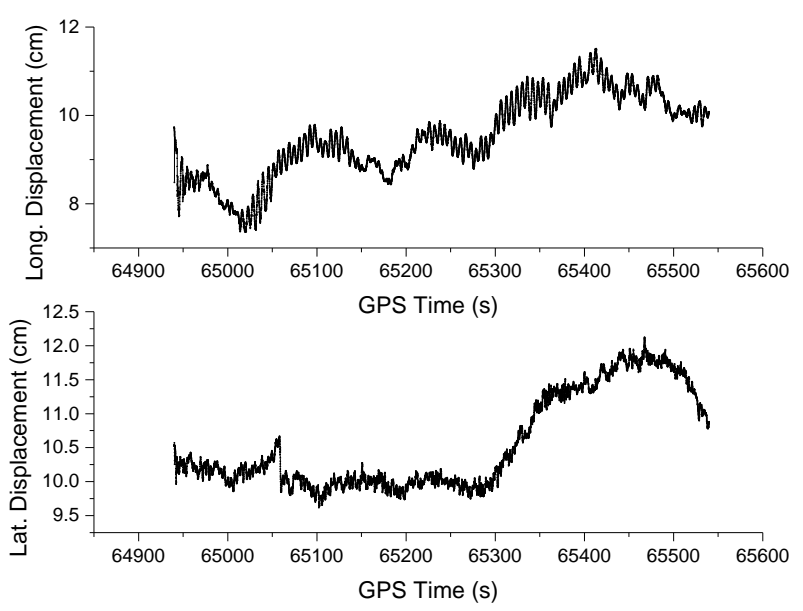

Fig. 15. Reconstructed bridge movements.

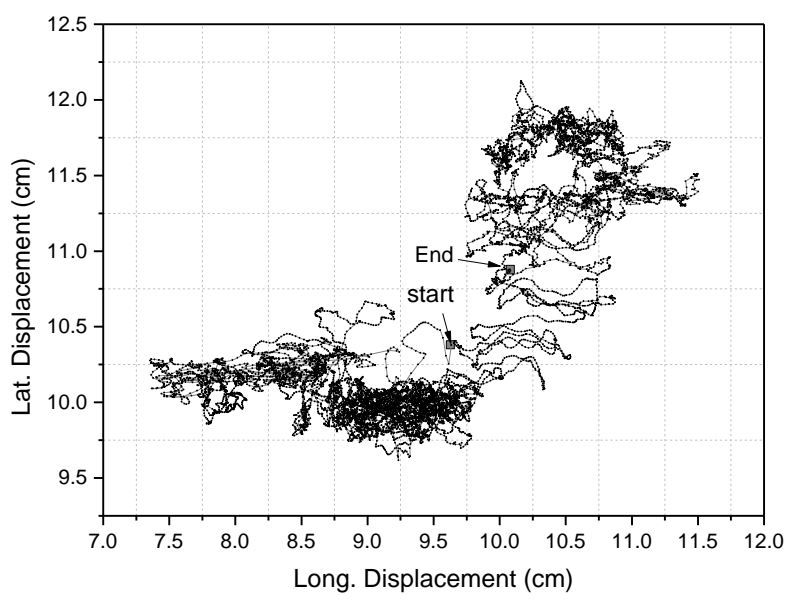

Fig. 16. Wind-induced responses of the bridge in horizontal direction.

\subsection{Spectral analysis and modal frequencies detection}

To obtain the natural frequencies of the long span bridge, a costeffective and widely used approach is to extract bridge dynamics under ambient loadings, mainly wind loadings for the bridge tower in our research. The existence of dominant frequencies in the time series was confirmed by the spectra analysis, the FFT algorithm and a frequency peak-picking approach were used to in the present study.

By analyzing the GPS displacement time series and acceleration time series, we can obtain the frequency spectrum of the wind-induced responses. Fig.17 shows the frequency spectrum for the longitudinal response, only the frequency characteristic above $0.1 \mathrm{~Hz}$ are shown in the figure. It can be seen that the identified lowest dominant frequency is $0.172 \mathrm{~Hz}$, which is detected from both GPS-derived displacements and accelerometer data series, and it is believed to be the first natural frequency of bridge tower. Generally, more frequency peaks less than 10 $\mathrm{Hz}$ (the Nyquist frequency of accelerometer data) can be detected from acceleration time series, it also can be seen that accelerometer data is contaminated by some measurement noise. Another frequency peak with smaller amplitude can also be detected from GPS-derived displacements, and the GPS time series are found to contain white noise and colored noise induced by measurement noise, multipath error and residual atmospheric error. On the other hand, some high-frequency characteristics cannot be detected from the integrated displacement time series, which is regarded to be caused by removal of low-amplitude acceleration during the displacement reconstruction operation.

Similar analysis is applied to lateral response measurements, the corresponding results are shown in Fig.18.It can be seen the first natural frequency cannot be detected from lateral GPS time series and acceleration time series, and higher frequency component identified by accelerometer is vanished in displacement time series, the higher frequency component extracted from the lateral acceleration time series shows an almost identical behavior as that of longitudinal acceleration time series. The modal frequencies detected with GPS and accelerometer under the wind loadings are listed in Table 1. 

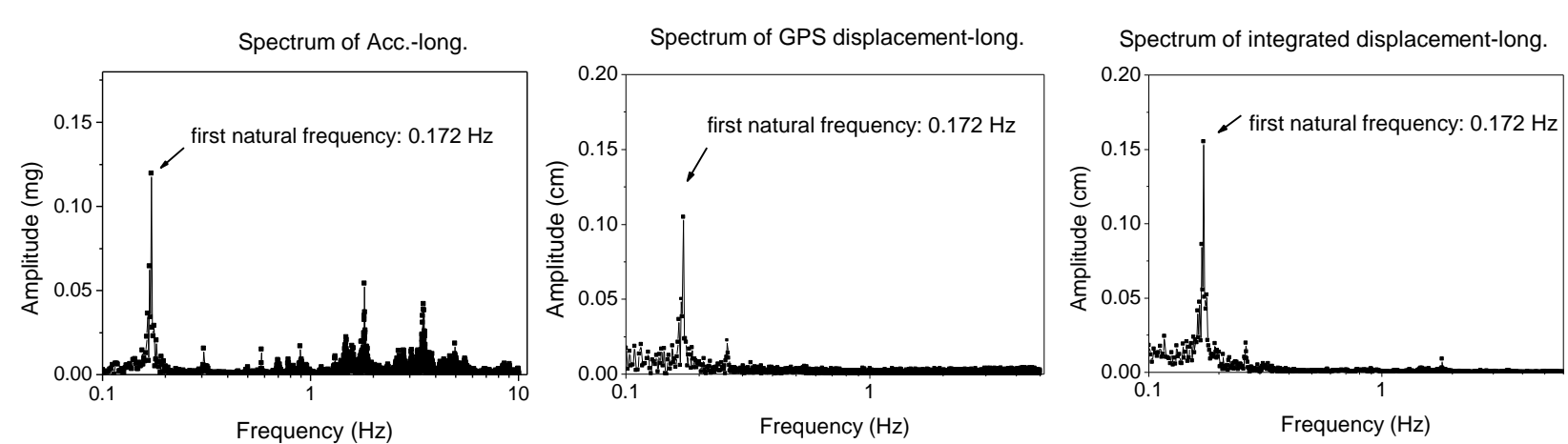

Fig. 17. Frequency spectrum from accelerometer (left), GPS (middle, high-frequency component) and integration deflection (right) in longitudinal direction.

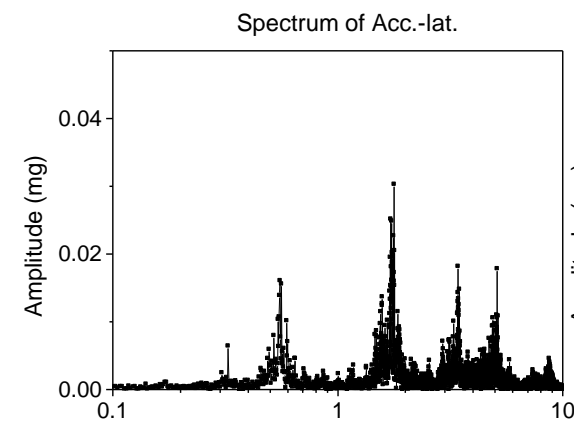

Frequency $(\mathrm{Hz})$

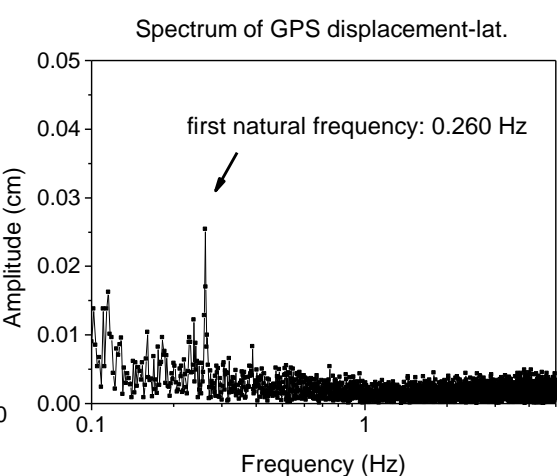

Frequency $(\mathrm{Hz})$

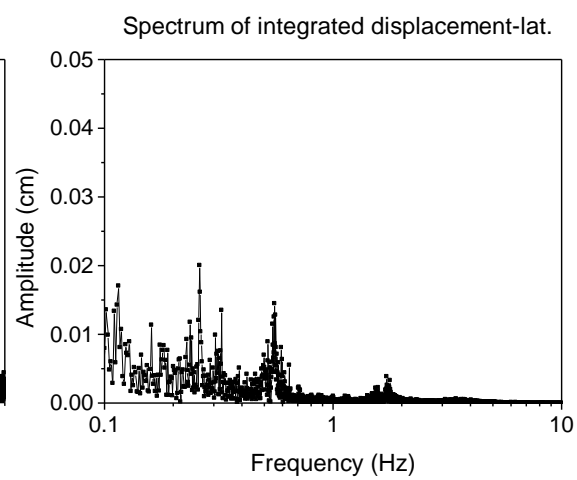

Fig. 18. Frequency spectrum from accelerometer (left), GPS (middle, high-frequency component) and integration deflection (right) in lateral direction.

Table 1Model frequencies detected from GPS and accelerometer measurements

\begin{tabular}{ccccccc}
\hline \multirow{2}{*}{$\begin{array}{c}\text { Model } \\
\text { Frequency }(\mathbf{H z})\end{array}$} & GPS & Acc. & Integration & GPS & Acc. & Integration \\
\cline { 2 - 7 } & 0.172 & 0.172 & 0.172 & - & - & 0.260 \\
2 & 0.260 & 0.307 & 0.260 & 0.260 & 0.325 & 0.325 \\
3 & - & 0.580 & - & - & 0.553 & 0.553 \\
4 & - & 1.810 & 1.810 & - & 1.775 & 1.775 \\
5 & - & 3.482 & - & - & 3.417 & - \\
6 & - & 4.943 & - & - & 5.097 & - \\
\hline
\end{tabular}

The linear amplitude spectra of longitudinal and lateral displacement time series for the whole time domain obtained from Morelet Wavelet transformation is shown in Fig. 19, the specific spectrum thus contain time information. It is evidently that largest amplitude are below 0.004
$\mathrm{Hz}$, this indicated the quasi-static movements are largely affected by strong winds. In the meantime, multipath effects can also be observed in the amplitude spectra, and the lower frequency multipath signature for the lateral displacements can be observed, while the dynamic response are not clearly shown in the figure due to frequency range limit.
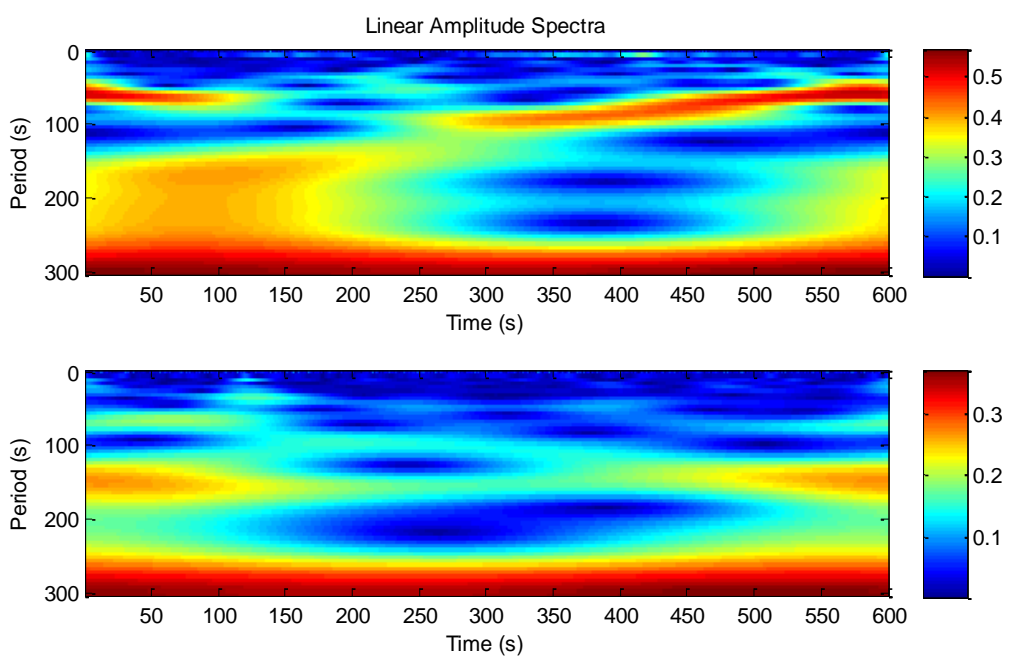
Fig. 19. Wavelet linear amplitude spectra of longitudinal(top) and lateral (bottom)GPS displacement.

\section{Discussions and Conclusions}

The aim of this study is to characterize the responses of a long span cable-stayed bridge under strong wind excitation, a GPS/accelerometer/anemometer integrated data processing technique, which is capable of providing accurate estimates of quasi-static movements and dynamic responses, is presented in this paper. The dynamics of bridge are extracted with a multi-step technique, including a frequency peak-picking approach based on FFT algorithm, noisy GPS displacement and acceleration de-noising technique based on Vondrak filter, followed by the low frequency noise elimination and displacement reconstruction scheme using noise-mitigated accelerations, the total displacement with improved measurement accuracy was reconstructed using the RLS filter.

A real-time GPS/accelerometer/anemometer integrated system has been installed on the Erqi Yangtze River Bridge for on-line monitoring of bridge deck and supporting tower movements, the data collected on the center supporting tower during strong winds are used to support the data analysis. It shows a highly correlation properties between the responses and wind speed, the results show clearly the GPS can measure the quasi-static movements, while less accurate high-frequency displacements which contaminated by measurement noise can be obtained. On the other hand, the displacements reconstructed from accelerometer can detect very small movements with the amplitude of a few millimeters, while it is difficult to measure the static component. The total horizontal displacements of the tower of bridge at millimeter accuracy are extracted using the integrated data processing scheme. The first natural frequency of $0.172 \mathrm{~Hz}$ is identified from the spectrum of GPS data, as validated by comparison with the spectrum of accelerometer data. However, higher frequencies are detectable with accelerometer. The results indicate a highly reliable estimate of displacement and natural frequency can be observed by the multi-sensor integration system, the integrated data processing technique will be beneficial to improve the overall monitoring performance.

\section{Conflict of interest}

The authors declare that there is no conflict of interests regarding the publication of this paper.

\section{Acknowledgements}

This work was partially supported by the Fundamental Research Funds for the Central Universities under grant number 2014ZDPY15, the Program for New Century Excellent Talents in University under grant number NCET-13-1019, a project funded by the Priority Academic Program Development of Jiangsu Higher Education Institutions, and the Cooperative Innovation Center of Jiangsu Province.

\section{References}

[1] Meng X. Real-time deformation monitoring of bridges using GPS/accelerometers: University of Nottingham; 2002.

[2] Meo M, Zumpano G, Meng X, Cosser E, Roberts G, Dodson A. Measurements of dynamic properties of a medium span suspension bridge by using the wavelet transforms. Mechanical systems and signal processing. 2006;20:1112-33.

[3] Psimoulis P, Pytharouli S, Karambalis D, Stiros S. Potential of Global Positioning System (GPS) to measure frequencies of oscillations of engineering structures. Journal of Sound and Vibration. 2008;318:606-23.

[4] Nickitopoulou A, Protopsalti K, Stiros S. Monitoring dynamic and quasi-static deformations of large flexible engineering structures with GPS: accuracy, limitations and promises. Engineering Structures. 2006;28:1471-82.
[5] Breuer P, Chmielewski T, Górski P, Konopka E, Tarczyński L. The Stuttgart TV Tower-displacement of the top caused by the effects of sun and wind. Engineering Structures. 2008;30:2771-81.

[6] Hristopulos D, Mertikas S, Arhontakis I, Brownjohn J. Using GPS for monitoring tallbuilding response to wind loading: filtering of abrupt changes and low-frequency noise, variography and spectral analysis of displacements. GPS Solutions. 2007;11:85-95.

[7] Moschas F, Stiros S. Measurement of the dynamic displacements and of the modal frequencies of a short-span pedestrian bridge using GPS and an accelerometer. Engineering Structures. 2011;33:10-7.

[8] Roberts GW, Cosser E, Meng X, Dodson A. High frequency deflection monitoring of bridges by GPS. Positioning. 2004;1:0.

[9] Yi T-H, Li H-N, Gu M. Experimental assessment of high-rate GPS receivers for deformation monitoring of bridge. Measurement. 2013;46:420-32.

[10] Kijewski-Correa T, Kareem A, Kochly M. Experimental verification and full-scale deployment of global positioning systems to monitor the dynamic response of tall buildings. Journal of Structural Engineering. 2006;132:1242-53.

[11] Moschas F, Stiros S. Noise characteristics of high-frequency, short-duration GPS records from analysis of identical, collocated instruments. Measurement. 2013;46:1488506.

[12] Genrich JF, Bock Y. Instantaneous geodetic positioning with 10-50 Hz GPS measurements: Noise characteristics and implications for monitoring networks. Journal of Geophysical Research: Solid Earth (1978-2012). 2006;111.

[13] Yu J, Meng X, Shao X, Yan B, Yang L. Identification of dynamic displacements and modal frequencies of a medium-span suspension bridge using multimode GNSS processing. Engineering Structures. 2014;81:432-43.

[14] Cocard M, Bourgon S, Kamali O, Collins P. A systematic investigation of optimal carrier-phase combinations for modernized triple-frequency GPS. Journal of Geodesy. 2008;82:555-64.

[15] Meng X. From Structural Health Monitoring to Geo-Hazard Early Warning: An Integrated Approach Using GNSS Positioning Technology. Earth Observation of Global Changes (EOGC): Springer; 2013. p. 285-93.

[16] Townsend B, Fenton P. A practical approach to the reduction of pseudorange multipath errors in a L1 GPS receiver. Proceedings of the 7th International Technical Meeting of the Satellite Division of the Institute of Navigation, Salt Lake City, UT, USA1994.

[17] Townsend B, Fenton P, Van Dierendonck K, Van Nee R. L1 carrier phase multipath error reduction using MEDLL technology. PROCEEDINGS OF ION GPS: INSTITUTE OF NAVIGATION; 1995. p. 1539-44.

[18] Han S, Rizos C. GPS multipath mitigation using FIR filters. Survey Review. 2000;35:487-98.

[19] Ge L, Han S, Rizos C. Multipath mitigation of continuous GPS measurements using an adaptive filter. GPS solutions. 2000;4:19-30.

[20] Zheng D, Zhong P, Ding X, Chen W. Filtering GPS time-series using a Vondrak filter and cross-validation. Journal of Geodesy. 2005;79:363-9.

[21] Kijewski-Correa T, Kochly M. Monitoring the wind-induced response of tall buildings: GPS performance and the issue of multipath effects. Journal of Wind Engineering and Industrial Aerodynamics. 2007;95:1176-98.

[22] Moschas F, Stiros S. Dynamic multipath in structural bridge monitoring: an experimental approach. GPS solutions. 2014;18:209-18.

[23] Meng X, Roberts G, Dodson A, Cosser E, Barnes J, Rizos C. Impact of GPS satellite and pseudolite geometry on structural deformation monitoring: analytical and empirical studies. Journal of Geodesy. 2004;77:809-22.

[24] Thong Y, Woolfson M, Crowe J, Hayes-Gill B, Jones D. Numerical double integration of acceleration measurements in noise. Measurement. 2004;36:73-92.

[25] Park K-T, Kim S-H, Park H-S, Lee K-W. The determination of bridge displacement using measured acceleration. Engineering Structures. 2005;27:371-8.

[26] Lee HS, Hong YH, Park HW. Design of an FIR filter for the displacement reconstruction using measured acceleration in low - frequency dominant structures. 
International Journal for Numerical Methods in Engineering. 2010;82:403-34.

[27] Hong YH, Kim H-K, Lee HS. Reconstruction of dynamic displacement and velocity from measured accelerations using the variational statement of an inverse problem. Journal of Sound and Vibration. 2010;329:4980-5003.

[28] Li X, Rizos C, Ge L, Tamura Y, Yoshida A. The complementary characteristics of GPS and accelerometer in monitoring structural deformation. Ion 2005 Meeting2005.

[29] Meng X, Dodson A, Roberts G. Detecting bridge dynamics with GPS and triaxial accelerometers. Engineering Structures. 2007;29:3178-84.

[30] Li X, Ge L, Ambikairajah E, Rizos C, Tamura Y, Yoshida A. Full-scale structural monitoring using an integrated GPS and accelerometer system. GPS solutions. 2006;10:233-47.

[31] Chan W, Xu Y, Ding X, Dai W. An integrated GPS-accelerometer data processing technique for structural deformation monitoring. Journal of Geodesy. 2006;80:705-19.

[32] Smyth A, Wu M. Multi-rate Kalman filtering for the data fusion of displacement and acceleration response measurements in dynamic system monitoring. Mechanical Systems and Signal Processing. 2007;21:706-23.

[33] Hwang J, Yun H, Park S-K, Lee D, Hong S. Optimal methods of RTK-
GPS/accelerometer integration to monitor the displacement of structures. Sensors. 2012;12:1014-34.

[34] Vondrak J. A contribution to the problem of smoothing observational data. Bulletin of the Astronomical Institutes of Czechoslovakia. 1969;20:349.

[35] Hong YH, Park HW, Lee HS. A regularization scheme for displacement reconstruction using acceleration data measured from structures. The 15th International Symposium on: Smart Structures and Materials \& Nondestructive Evaluation and Health Monitoring: International Society for Optics and Photonics; 2008. p. 693228--11.

[36] Tikhonov AN, Arsenin VIAk, John F. Solutions of ill-posed problems: Winston Washington, DC; 1977.

[37] Hansen PC, O'Leary DP. The use of the L-curve in the regularization of discrete illposed problems. SIAM Journal on Scientific Computing. 1993;14:1487-503.

[38] Vaseghi SV. Advanced digital signal processing and noise reduction: John Wiley \& Sons; 2008.

[39] Farhang-Boroujeny B. Adaptive filters: theory and applications: John Wiley \& Sons; 2013. 